Article

\title{
Fremy's Salt-Mediated Oxidative Addition. A New Approach in the Total Synthesis of Naturally Dipetalolactone and Its Immunomodulatory Activity
}

\author{
Yasser Selim ${ }^{1, *}$ Nabil Ouf ${ }^{2,3}$ and Mohamed Sakran ${ }^{2,4}$ \\ 1 Faculty of Specific Education, Zagazig University, Zagazig 44519, Egypt \\ 2 Department of Chemistry and Biochemistry, Faculty of Science, Tabuk University, \\ Tabuk PO Box 699, Saudi Arabia \\ 3 Department of Chemistry, Faculty of Science, Zagazig University, Zagazig 44519, Egypt \\ 4 Department of Chemistry, Faculty of Science, Tanta University, Tanta 31527, Egypt \\ * Author to whom correspondence should be addressed; E-Mail: y2selem@yahoo.com; \\ Tel.: +20-55-238-6547; Fax: +20-55-234-5515.
}

Received: 2 July 2013; in revised form: 30 July 2013 / Accepted: 26 August 2013 / Published: 16 September 2013

\begin{abstract}
The structure of the natural dipyranocoumarin dipetalolactone has been confirmed by an unambiguous synthetic route from resorcinol. This sequence was initiated by a pyran ring formation step which introduced the 3-chloro-3-methylbut-1-yne moiety. Then, the expected product undergoes a Fremy's salt-meditated oxidative addition followed by ring closure to yield dipetalolactone. Dipetalolactone was also found to have immunological activity in a mouse carcinoma S180-bearing mice cell line.
\end{abstract}

Keywords: coumarins; dipyranocoumarin; dipetalolactone; Fremy's salt

\section{Introduction}

Dipetalolactone is a natural product dipyranocoumarin isolated from different plants like Zanthoxylum dipetalum, Metrodorea flavido and Diplolaene mollis (Rutaceae) [1]. The dipyranocoumarins, a group of natural products from several tropical plants of the genus Calophyllum are characterized by coumarin, chromene and chromane ring systems [2]. Biologically, dipyranocoumarins are very useful and many of them have exhibited anti HIV, antibacterial, antitumor, vasodilator (in coronary vessels) and anticoagulant activities [3]. It was long noted that 
most coumarins are free from toxic side effects and may be given for years without side effects; overdoses, however, may causes hemorrhages [3]. Coumarins are widespread in the Angiosperms but they are rather rare in Gymnosperms and lower plants. They present great structural variety, especially in the Apiaceae and Rutaceae, and are additionally found in many other plants families like the Asteraceae, Poaceae and Rubiaceae [4]. The family Rutaceae belongs to the order Rutales characterized by the occurrence of coumarins in all its families. Coumarins, although very frequent in the family as a whole, are confined to four sub-families (Aurantioideae, Flindersioideae, Toddalioideae and Rutoideae). The genus Zanthoxylum in the subfamily Rutoideae is characterized by the presence of different types of coumarins (simple, linear, dihydrofurocoumarins, furocoumarins and pyranocoumarins). The linear and angular dihydrofurocoumarins and precursors have been identified in several species of the genus, but angular dihydrofurocoumarins are not common in other species of the family Rutaceae, so it can be of chemotaxonomic value for the genus Zanthoxylum [5].

\section{Results and Discussion}

\subsection{Chemistry}

Previously dipetalolactone has been prepared from 5,7-dihydroxycoumarin [6]. In the context of this work, we have synthesized dipetalolactone starting from resorcinol using Fremy's salt as meditating oxidizing agent. To our knowledge, except for the reaction of compound 1 with 3-chloro3-methylbut-1-yne [6], there has not been any work in the same direction. Our studies were initiated by the reaction of resorcinol 1 and 3-chloro-3-methyl but-1-yne, which introduces the 3-methylbut-1-enyl group exclusively and in high yield at the $\mathrm{OH}$ of $\mathrm{C} 1$ to form 3-(2-methylbut-3-yn-2-yloxy)phenol (2) followed by reflux with xylene for $8 \mathrm{~h}$ to give pyran ring 3 (Scheme 1). The ${ }^{1} \mathrm{H}-\mathrm{NMR}$ of compound 3 indicates the presence of five aromatic protons at $\delta 6.1(\mathrm{~d}, 1 \mathrm{H}, J=9.1 \mathrm{~Hz}, \mathrm{H}-3), 6.8(\mathrm{~d}, 1 \mathrm{H}, J=9.4 \mathrm{~Hz}$, $\mathrm{H}-4), 6.22(\mathrm{~d}, 1 \mathrm{H}, J=8.1 \mathrm{~Hz}, \mathrm{H}-6), 7.1(\mathrm{~m}, 1 \mathrm{H}, J=8.4 \mathrm{~Hz}, \mathrm{H}-7)$ and $6.13(\mathrm{~d}, 1 \mathrm{H}, J=8.5 \mathrm{~Hz}, \mathrm{H}-8)$. Further the ${ }^{1} \mathrm{H}-\mathrm{NMR}$ spectrum displayed signals of two methyl at $\delta 1.55(\mathrm{~s}, 3 \mathrm{H})$ and $1.65(\mathrm{~s}, 3 \mathrm{H})$. Compound 3 was oxidized when a solution of 3 is treated with a solution of $\mathrm{KH}_{2} \mathrm{PO}_{4}$ in water and $\left(\mathrm{KSO}_{3}\right)_{2} \mathrm{NO}$ at $0{ }^{\circ} \mathrm{C}$ to give 2,2-dimethyl 2-H-chromene-5,8-dione (4). Fremy's salt oxidizes most phenols to $p$-quinones when there is no Para-substituent, whereby oxygen incorporation occurs exclusively from the oxygen of Fremy's salt on the phenol group. Compound 4 was characterized by its ${ }^{13} \mathrm{C}-\mathrm{NMR}$ spectrum which revealed the presence of two signals at $\delta 185.5$ (C-1) and 181.7 (C-2) respectively, which indicated the presence of a Para-diketone structure. The quinoid structure constitutes one of the most interesting classes of compounds in Organic Chemistry [7]. The chemistry of quinones is largely dependent on the substituent being either on the quinonic or on adjacent rings. This is reflected in their chemical reactivity, especially in heterocyclic quinones [8]. Hydroxylated quinones that have one or more hydroxy groups attached directly to the quinone moiety are found in Nature in great variety. A method of broad applicability for the preparation of the hydroxy quinone moiety is through Thiele-Winter acetoxylation. The method involves the reaction of 1,4- or 1,2-quinone derivatives with acetic anhydride. Thus, the conversion of the Para-quinoid structure to the corresponding triacetate derivative occurred by treating it with acetic anhydride in dioxane [9,10] to give the expected product 5. The triacetoxy derivatives - isolated in fair to excellent yields - are 
hydrolyzed to the corresponding hydroxyhydroquinone derivatives under either acidic or basic conditions. The latter, as a rule without isolation, are then oxidized to the desired hydroxy quinone compounds. In many cases, especially under basic conditions, the oxidation also proceeds with atmospheric oxygen [9]. Treatment of compound 5 with hydrochloric acid in the presence of ferric chloride gave the expected ortho-hydroxy quinone structure 6 in $88 \%$ yield. The di pyrano derivative 7 was also obtained by refluxing 6 with 3-chloro-3-methylbut-1-yne, followed by ring closure by refluxing the product in dry xylene to give compound $\mathbf{8}$. Compound 7 was characterized by ${ }^{1} \mathrm{H}-$ and ${ }^{13} \mathrm{C}$-NMR spectroscopy which revealed the presence of signals at $\delta 4.07$ as a sharp singlet, i.e., that is flanked between two ketone groups and two aromatic protons at $\delta 5.84$, and the absence of signals of the hydroxyl group proton at 6.82. Also, the IR spectrum showed the absence of a hydroxyl group peak, thus confirming the formation of 7-hydroxyl-2,2-dimethyl 2-H-chromene-5,7,8(6H)-trione (7). Finally the dipyranocoumarin was prepared by Wittig reaction in the presence of clay (montmorillonite KSF) and compared with an authentic sample of dipetalolactone [6]. The UV absorption in ethanol exhibited peaks at $\lambda_{\max }{ }^{\mathrm{MeoH}}: 282(\log \varepsilon 3.75), 232(\log \varepsilon$ 3.97) and 211 (log $\varepsilon 4.27)$ characteristic of dipyranocoumarins. The IR spectral band at $1,720 \mathrm{~cm}^{-1}$ indicates the presence of a $\delta$-lactone. It was also characterized by its ${ }^{1} \mathrm{H}$ - and ${ }^{13} \mathrm{C}$-NMR spectra which revealed the absence of acetyl signals, thus confirming the cyclization and formation of dipetalolactone (10).

Scheme 1. Total synthesis of natural dipetalolactone.
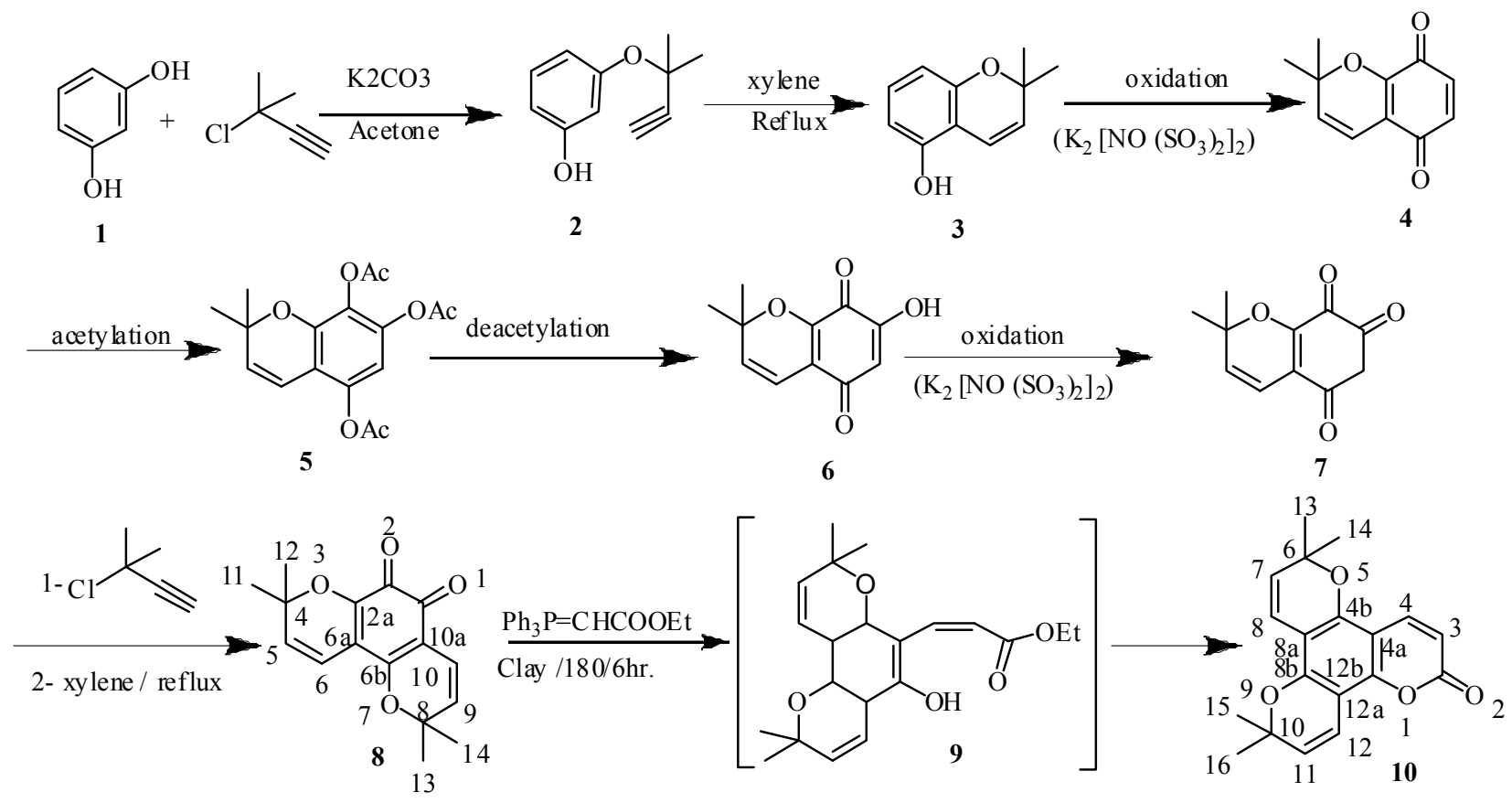

\subsection{Biological Activity}

\subsubsection{Effects of Compound $\mathbf{1 0}$ on Body and Tumor Weights of S180-Bearing Mice}

All the tumor-injected mice survived following the treatments with water or any of the four doses of compound 10 until they were sacrificed for analysis of immunological features. When the tumor masses were removed, we observed that the animals treated with compound $\mathbf{1 0}$ had no significant 
changes in body and tumor weight (Table $1, p>0.05$ ), but showed lower tumor growth to a certain extent compared with the S180 control group. Higher concentrations appeared to show more effective inhibition. Complete regression of tumor was not observed in any group.

Table 1. Effects of compound $\mathbf{1 0}$ on body and tumor weights of S180-bearing mice.

\begin{tabular}{cccccc}
\hline Group & $\begin{array}{c}\text { Concentration } \\
(\mathbf{m g} / \mathbf{k g})\end{array}$ & $\begin{array}{c}\text { Body weight } \\
(\mathbf{g}) \text { at begin }\end{array}$ & $\begin{array}{c}\text { Body weight } \\
(\mathbf{g}) \text { at end }\end{array}$ & $\begin{array}{c}\text { Tumor weight } \\
(\mathbf{g})\end{array}$ & $\begin{array}{c}\text { Inhibition } \\
(\mathbf{\%})\end{array}$ \\
\hline Normal control & & $25.5 \pm 1.5$ & $33.4 \pm 3.55$ & & - \\
S180 control & & $25.8 \pm 1.35$ & $32.6 \pm 3.42$ & $4.6 \pm 1.2$ & - \\
& 50 & $25.6 \pm 1.44$ & $32.8 \pm 2.55$ & $4.22 \pm 1.11$ & 2.2 \\
Compound 10 & 100 & $25.1 \pm 1.12$ & $33.0 \pm 3.22$ & $3.32 \pm 0.85$ & 6.1 \\
& 250 & $25.7 \pm 1.4$ & $33.8 \pm 3.24$ & $3.77 \pm 0.92$ & 12.5 \\
& 500 & $25.33 \pm 1.35$ & $33.4 \pm 3.15$ & $3.66 \pm 0.62$ & 22 \\
\hline
\end{tabular}

2.2.2. Effects of Compound $\mathbf{1 0}$ on Immune Functions of S180-Bearing Mice

\subsubsection{Effects of Compound $\mathbf{1 0}$ Extract on Humoral Immune Function}

The effect of compound $\mathbf{1 0}$ on humoral immune function was estimated by measuring quantitative hemolysis of sheep red blood cells in vivo (Table 2). All dose treatments could markedly increase the antibodies secreted by spleen cells in mice, where $500 \mathrm{mg} / \mathrm{kg}$ dose was the best as a typical immunostimulator was used.

Table 2. Determination of $\mathrm{LD}_{50}$ of compound $\mathbf{1 0}$ given i.p. in adult mice.

\begin{tabular}{cccccc}
\hline Group & $\begin{array}{c}\text { Concentration } \\
(\mathbf{m g} / \mathbf{k g})\end{array}$ & $\begin{array}{c}\text { Quantitative hemolysis } \\
\text { of sheep red blood cells } \\
\left(\mathbf{H C}_{\mathbf{5 0}}\right)\end{array}$ & $\begin{array}{c}\text { Lymphocyte } \\
\text { proliferation } \\
\mathbf{( c p m )}\end{array}$ & $\begin{array}{c}\text { NK cytotoxic } \\
\text { activity (\%) }\end{array}$ & $\begin{array}{c}\text { Phagocytosis } \\
\text { rate }\end{array}$ \\
\hline Normal control & - & $450 \pm 24.1 * * *$ & $12,530 \pm 4,121 * *$ & $58.4 \pm 1.22 *$ & $43.1 \pm 2.15 * * *$ \\
S180 control & - & $60.11 \pm 3.32$ & $2,822 \pm 881$ & $48.6 \pm 1.33$ & $35.0 \pm 3.31$ \\
& 50 & $120 \pm 9.8 * * *$ & $3,251 \pm 1,161$ & $50.2 \pm 2.51$ & $37.8 \pm 2.31 *$ \\
Compound 10 & 100 & $221 \pm 10.4 * * *$ & $5,322 \pm 1,131 * *$ & $55.8 \pm 2.64 * * *$ & $39.9 \pm 3.55 * * *$ \\
& 250 & $232 \pm 11.2 * * *$ & $6,455 \pm 1,228 *$ & $56.6 \pm 2.55 * * *$ & $41.12 \pm 2.34 * *$ \\
& 500 & $243 \pm 10.5 * * *$ & $7,220 \pm 2,122 *$ & $58.6 \pm 3.1 * * *$ & $42.3 \pm 3.21 * *$ \\
\hline
\end{tabular}

Values are mean \pm S.D. of 10 mice; * Significantly different from S180 control group at $p<0.05$;

** Significantly different from S180; *** Significantly different from S180 control group at $p<0.001$.

\subsubsection{Effects of Compound $\mathbf{1 0}$ on Cellular Immune Function}

The effect of compound $\mathbf{1 0}$ on cellular immune function was estimated by measuring lymphocyte proliferation and Natural Killer (NK) cell cytotoxicity in vivo. In the assay, spleen lymphocyte proliferation and NK activity were significantly decreased in the S180 control group. Compound $\mathbf{1 0}$ at doses at 100, 250 and $500 \mathrm{mg} / \mathrm{kg}$ demonstrated remarkable spleen lymphocyte proliferation stimulation and increased NK activity, whereas the dose of $50 \mathrm{mg} / \mathrm{kg}$ did not (Table 2). In the NK cell cytotoxicity assay, additionally, the same doses of compound $\mathbf{1 0}$ could significantly regulate cellular immunity close to a normal level. 


\subsubsection{Effects of Compound $\mathbf{1 0}$ on Nonspecific Immune Function}

This effect of compound $\mathbf{1 0}$ was estimated by measuring the phagocytic activity of peritoneal macrophages in vivo. Administration of compound $\mathbf{1 0}$ could significantly enhance the phagocytic activity of peritoneal macrophages at all doses compared to the untreated group (Table 2). The doses at $100,250 \mathrm{mg} / \mathrm{kg}$ and $500 \mathrm{mg} / \mathrm{kg}$ showed the most effective activity, which was close to that of the normal control.

\section{Experimental}

\subsection{General}

All melting points were taken on an Electro thermal IA9000 series digital melting point apparatus. Elemental analysis data were obtained from the Micro analytical unit, Cairo University, Cairo. Egypt. The IR spectra $(\mathrm{KBr})$ were recorded on the Elmer model 1430 spectrophotometer. ${ }^{1} \mathrm{H}$ - and ${ }^{13} \mathrm{C}-\mathrm{NMR}$ spectra were recorded in $\mathrm{CDCl}_{3}$ on a Bruker Avance DRX-500 spectrometer $\left({ }^{1} \mathrm{H}\right.$ at $500 \mathrm{MHz}$ and ${ }^{13} \mathrm{C}$ at $125 \mathrm{MHz}$ ). ESIMS and HRESIMS experiments were performed using a Micromass Q-TOF (Manchester, UK). TLC was carried out on precoated silica gel $60 \mathrm{~F}_{254}$ (Merck, Munich, Germany) and spots were visualized by UV lamb. Column chromatography was carried out on silica gel 60 (63-200 $\mu \mathrm{m}$, Merck).

\subsection{3-(2-Methylbutyl-3-yn-2-yloxy)phenol (2)}

To a solution of resorcinol $(0.01 \mathrm{~mol})$ and potassium carbonate $(0.01 \mathrm{~mol})$ in acetone $(30 \mathrm{~mL})$, 3-chloro-2-methylbut-1-yne $(0.01 \mathrm{~mol})$ was added drop wise under nitrogen. The reaction mixture was heated and stirred at $70{ }^{\circ} \mathrm{C}$ for $72 \mathrm{~h}$ and monitored by TLC. After cooling to room temperature the solution was poured in water $(100 \mathrm{~mL})$ then acidified with dilute $\mathrm{HCl}(5 \%)$ to remove the residue of $\mathrm{K}_{2} \mathrm{CO}_{3}$ and extracted by diethyl ether $(4 \times 15 \mathrm{~mL})$. The mixture was dried over anhydrous sodium sulphate, filtered and then the solvent was evaporated and the crude product was purified by column chromatography over silica gel (100-200 mesh) and eluted with mixtures of hexane/ethyl acetate $(1: 1 \mathrm{v} / \mathrm{v})$ as eluent to give compound 2 , which was crystallized from dichloromethane/hexane as solvent system to give pale white crystals $(40 \%)$ with mp $160-162{ }^{\circ} \mathrm{C}$; IR (KBr) $v_{\max } \mathrm{cm}^{-1}: 3,540$, 3,352, 1,335, 1,119, 921, 849; ${ }^{1} \mathrm{H}-\mathrm{NMR}\left(\mathrm{CDCl}_{3}\right): \delta 7.18(\mathrm{Ar}-1 \mathrm{H}, \mathrm{m}, J=9.2 \mathrm{~Hz}, \mathrm{H}-5), 6.58(\mathrm{Ar}-1 \mathrm{H}, \mathrm{m}$, $J=9.1 \mathrm{~Hz}, \mathrm{H}-4), 6.51(\mathrm{Ar}-1 \mathrm{H}, \mathrm{m}, J=8.9 \mathrm{~Hz}, \mathrm{H}-2,6), 3.51(1 \mathrm{H}, \mathrm{s}, \equiv \mathrm{CH}), 1.69\left(6 \mathrm{H}, \mathrm{s}, 2 \mathrm{CH}_{3}\right)$; ${ }^{13} \mathrm{C}-\mathrm{NMR}\left(\mathrm{CDCl}_{3}\right): 160.1(\mathrm{C}-3), 157.4(\mathrm{C}-1), 130.5(\mathrm{C}-5), 107.8(\mathrm{C}-4), 102.7(\mathrm{C}-2), 85.5(\underline{\mathrm{C}} \equiv \mathrm{CH})$, $81.7\left[\underline{\mathrm{C}}\left(\mathrm{CH}_{3}\right)_{2}\right], 75.4(\equiv \mathrm{CH}), \delta 29.2\left(2 \mathrm{CH}_{3}\right)$; EIMS $\left(\mathrm{C}_{11} \mathrm{H}_{12} \mathrm{O}_{2}\right)(\%$ int. $) \mathrm{m} / z 176(100), 205[\mathrm{M}-\mathrm{H}]^{+}$ (25), $190[\mathrm{M}-\mathrm{OH}]^{+}(21), 176\left[\mathrm{M}-2 \mathrm{CH}_{3}\right]^{+}(8)$ and $107\left[\mathrm{M}-\mathrm{HC} \equiv \mathrm{C}-\mathrm{C}\left(\mathrm{CH}_{3}\right)_{2}-\mathrm{O}\right]^{+}(12)$; Analysis: calcd. $\% \mathrm{C}, 74.98 ; \% \mathrm{H}, 6.83 ; \% \mathrm{O}, 18.18$. Found: $\% \mathrm{C} 74.88 ; \% \mathrm{H}, 6.81 ; \% \mathrm{O} 18.09$.

\subsection{2,2-Dimethyl-2H-chromen-5-ol (3)}

Compound 2 (0.01 mole) was boiled in dry xylene for $8 \mathrm{~h}$ under a nitrogen atmosphere, the reaction mixture was cooled, and then extracted with dichloromethane. The residue after removing the solvent was subjected to column chromatography over silica gel $(800 \mathrm{~g})$ and eluted with mixtures of 
hexane/ethyl acetate $(1: 2 \mathrm{v} / \mathrm{v})$ as eluent to produce compound 3, which was crystallized from ethanol to give white crystals $(60 \%)$ with $\mathrm{mp} 155-156{ }^{\circ} \mathrm{C}$; IR $(\mathrm{KBr}) v_{\max } \mathrm{cm}^{-1}: 3,540,3,352,1,335,1,129$, 931, 841; ${ }^{1} \mathrm{H}-\mathrm{NMR}\left(\mathrm{CDCl}_{3}\right): \delta 11.2$ (s, OH exchangeable with $\left.\mathrm{D}_{2} \mathrm{O}\right), 7.13(\mathrm{Ar}-1 \mathrm{H}, \mathrm{m}, \mathrm{H}-7), 6.86$ (Ar-1H, m, $J=9.2 \mathrm{~Hz}, \mathrm{H}-4), 6.55$ (Ar-1H, d, $J=8.9 \mathrm{~Hz}, \mathrm{H}-8), 6.31$ (Ar-1H, d, $J=8.9 \mathrm{~Hz}, \mathrm{H}-6$ ), 5.91 $(\mathrm{Ar}-1 \mathrm{H}, \mathrm{d}, J=9.1 \mathrm{~Hz}, \mathrm{H}-3), 1.59\left(3 \mathrm{H}, \mathrm{s}, 2 \mathrm{CH}_{3}\right) ;{ }^{13} \mathrm{C}-\mathrm{NMR}\left(\mathrm{CDCl}_{3}\right): \delta 156.7$ (C-5), $155.5(\mathrm{C}-8 \mathrm{a})$, 130.4 (C-7), 128.5 (C-3), 116.2 (C-4), 109.8 (C-4a), 109.7 (C-6), 106.5 (C-8), 85.7 (C-2), $28.5\left(2 \mathrm{CH}_{3}\right.$, C-9,10); EIMS $\left(\mathrm{C}_{11} \mathrm{H}_{12} \mathrm{O}_{2}\right)$ (\% int.) $m / z 176(100), 175[\mathrm{M}-\mathrm{H}]^{+}(32), 160[\mathrm{M}-\mathrm{OH}]^{+}(21)$ and 146 $\left[\mathrm{M}-2 \mathrm{CH}_{3}\right]^{+}(12)$; Analysis: calcd. $\% \mathrm{C}, 74.96 ; \% \mathrm{H}, 6.85 ; \% \mathrm{O}, 18.19$. Found: $\% \mathrm{C} 74.91 ; \% \mathrm{H}, 6.82$; $\% \mathrm{O} 18.12$.

\subsection{2,2-Dimethyl-2H-chromene-5,8-dione (4)}

To a solution of $\mathrm{KH}_{2} \mathrm{PO}_{4}(10 \mathrm{~g})$ in water $(200 \mathrm{~mL})$ was mechanically stirred in a $2 \mathrm{~L}$ round bottom flask and ice $(200 \mathrm{~g})$ was added compound $3(0.01 \mathrm{~mol})$. The flask was cooled in an ice-ethanol mixture and $\left(\mathrm{KSO}_{3}\right)_{2} \mathrm{NO}(6 \mathrm{~g}, 22.4 \mathrm{mmol})$ added, followed by a solution of 4-(methylmercapto)phenol $(1.0 \mathrm{~g}, 7.1 \mathrm{mmol})$ in diethyl ether $\left(20 \mathrm{~cm}^{3}\right)$. After $1 \mathrm{~min}$ the solution turned orange and after 5 min dark red. After $1 \mathrm{~h}$ the mixture was evaporated at $20{ }^{\circ} \mathrm{C}$ to remove the diethyl ether and extracted with chloroform $\left(3 \times 50 \mathrm{~cm}^{3}\right)$. Evaporation gave a crimson solid $(0.95 \mathrm{~g}, 86 \%$ yields $)$ with $\mathrm{mp} 180-181{ }^{\circ} \mathrm{C}$; IR (KBr) $v_{\max } \mathrm{cm}^{-1}: 1,750,1,735,1,343,1,139,951,841 ;{ }^{1} \mathrm{H}-\mathrm{NMR}\left(\mathrm{CDCl}_{3}\right): \delta 6.93(\mathrm{Ar}-1 \mathrm{H}, \mathrm{d}, \mathrm{H}-6,7)$, $6.44(\mathrm{Ar}-1 \mathrm{H}, \mathrm{s}, \mathrm{H}-4), 5.81(\mathrm{Ar}-1 \mathrm{H}, \mathrm{d}, J=9.2 \mathrm{~Hz}, \mathrm{H}-3), 1.42\left(3 \mathrm{H}, \mathrm{s}, 2 \mathrm{CH}_{3}\right) ;{ }^{13} \mathrm{C}-\mathrm{NMR}\left(\mathrm{CDCl}_{3}\right): \delta 185.5$ (C-5), 181.7 (C-8), 150.4 (C-8a), 136.2 (C-6), 135.5 (C-7), 132.8 (C-3), 119.7 (C-4a), 116.5 (C-4), 85.9 (C-2), $28.7\left(2 \mathrm{CH}_{3}, \mathrm{C}-9,10\right)$; EIMS $\left(\mathrm{C}_{11} \mathrm{H}_{10} \mathrm{O}_{3}\right)\left(\%\right.$ int.) $\mathrm{m} / z 190$ (100), $175\left[\mathrm{M}-1 \mathrm{CH}_{3}\right]^{+}$(22), $160\left[\mathrm{M}-2 \mathrm{CH}_{3}\right]^{+}(9), 162[\mathrm{M}-\mathrm{CO}](41)$; Analysis: calcd. $\% \mathrm{C}, 69.46 ; \% \mathrm{H}, 5.30 ; \% \mathrm{O}, 25.22$. Found: $\% \mathrm{C} 69.41 ; \% \mathrm{H}, 5.22 ; \% \mathrm{O} 25.12$.

\subsection{2,2-Dimethyl-2H-chromene-5,7,8-triyltriacetate (5)}

Acetylation was carried out following literature method [5]. In brief, compound 4 (0.01 mol) was dissolved in refluxing $p$-dioxane $(200 \mathrm{~mL})$ and acetic anhydride $(0.03 \mathrm{~mol})$ for $8 \mathrm{~h}$. After cooling, the acetylated compound solution was concentrated and dried under reduced pressure. The crude acetylated lignin was dissolved in chloroform $(3 \mathrm{~mL})$ and the solution was added with stirring to diethyl ether. The precipitated acetate was centrifuged, collected, and dried under high vacuum for $24 \mathrm{~h}$. The precipitate formed after cooling the mixture on ice water solution was recrystallized from ethanol give compound 5 as white crystals $(0.61 \mathrm{~g}, 50 \%)$ with $\mathrm{mp} 277-279{ }^{\circ} \mathrm{C}$. IR (KBr) $v_{\max } \mathrm{cm}^{-1}$ : 1,763, 1,750 and 1,710 (3 $\mathrm{C}=\mathrm{O}$ ester), 1,576, 1,345, 1,129, 911, 849; ${ }^{1} \mathrm{H}-\mathrm{NMR}\left(\mathrm{CDCl}_{3}\right): \delta 6.88$ $($ Ar-1H, d, $J=9.9 \mathrm{~Hz}, \mathrm{H}-4), 6.42(\mathrm{Ar}-1 \mathrm{H}, \mathrm{d}, J=9.1 \mathrm{~Hz}, \mathrm{H}-6), 5.88$ (Ar-1H, d, $J=8.5 \mathrm{~Hz}, \mathrm{H}-3$ ), 2.28, 2.2, $2.1\left(\mathrm{~s}, 9 \mathrm{H}, 3 \mathrm{C}_{3} \mathrm{CH}_{2} \mathrm{O}\right), 1.49\left(6 \mathrm{H}, \mathrm{s}, 2 \mathrm{CH}_{3}\right) ;{ }^{13} \mathrm{C}-\mathrm{NMR}\left(\mathrm{CDCl}_{3}\right): \delta 169.1(3 \mathrm{C}=\mathrm{O}), 153.2$ (C-8a), 147.2 (C-5), 144.2 (C-7), 130.1 (C-8), 127.4 (C-3), 114.1 (C-4a), 116.5 (C-4), 103.5 (C-6), 86.7 (C-2), $28.2\left(2 \mathrm{CH}_{3}, \quad \mathrm{C}-9,10\right), 20.5 \quad\left(3 \mathrm{CH}_{3}\right.$-ester); EIMS $\left(\mathrm{C}_{17} \mathrm{H}_{18} \mathrm{O}_{7}\right) \quad(\%$ int. $) \mathrm{m} / z \quad 334$ (100), $319\left[\mathrm{M}-1 \mathrm{CH}_{3}\right]^{+}(34), 304\left[\mathrm{M}-2 \mathrm{CH}_{3}\right]^{+}(21)$ and 199 [M-3OAc] (14)"; Analysis: calcd. \%C, 61.08; $\% \mathrm{H}, 5.43 ; \% \mathrm{O}, 31.48$. Found: \%C 60.88; \%H, 5.31; \%O 31.59. 


\subsection{7-Hydroxyl-2,2-dimethyl-2H-chromene-5,8-dione (6)}

Compound 6 was obtained by dissolving $(0.1 \mathrm{~mol})$ of compound 5 in $10 \% \mathrm{NaOH}$ solution, then by addition of $\mathrm{HCl}$, we obtain a precipitate, which is filtered off , then transferred into a conical flask and water $(30 \mathrm{~mL})$ is added. The mixture is brought to its boiling point, $\mathrm{FeCl}_{3}(1.5 \mathrm{~g})$ is added and boiled for $10 \mathrm{~min}$, the hot solution is filtered, washed with boiling water and he precipitate formed dried in air. Recrystallization from toluene gives brown crystals $(40 \%)$ with $\mathrm{mp} 254-255^{\circ} \mathrm{C}$; $\mathrm{UV}(\mathrm{MeOH}) \lambda_{\max }$ : $331\left(\log \varepsilon\right.$ 3.67), $292(\log \varepsilon 4.07), 262(\log \varepsilon 4.45) ; \mathrm{IR}(\mathrm{KBr}) v_{\max } \mathrm{cm}^{-1}: 3,340,1,752,1,722,1,576$, 1,345, 1,129, 911, 849; ${ }^{1} \mathrm{H}-\mathrm{NMR}\left(\mathrm{CDCl}_{3}\right): \delta 16.2(1 \mathrm{H}, \mathrm{s}, 1 \mathrm{OH}), 6.83(\mathrm{Ar}-1 \mathrm{H}, \mathrm{d}, J=9.1 \mathrm{~Hz}, \mathrm{H}-2), 6.42$ (Ar-1H, d, $J=9.2 \mathrm{~Hz}, \mathrm{H}-8), 6.81$ (Ar-1H, s, H-6), 6.41 (Ar-1H, d, $J=9.2 \mathrm{~Hz}, \mathrm{H}-4), 5.81$ (Ar-1H, d, $J=8.4 \mathrm{~Hz}, \mathrm{H}-3), 1.39(6 \mathrm{H}, \mathrm{s}, \mathrm{H}-10,11) ;{ }^{13} \mathrm{C}-\mathrm{NMR}\left(\mathrm{CDCl}_{3}\right): \delta 182.5$ (C-5), $196.2(\mathrm{C}-8), 176.5(\mathrm{C}-7)$, 141.2 (C-8a), 126.5 (C-3), 117.4 (C-4a), 115.5 (C-4), 109.5 (C-6), 86.7 (C-2), 29.5 (2CH3, C-9,10); EIMS $\left(\mathrm{C}_{11} \mathrm{H}_{10} \mathrm{O}_{4}\right)\left(\%\right.$ int.) $m / z 206(100), 205[\mathrm{M}-\mathrm{H}]^{+}(21), 190[\mathrm{M}-\mathrm{OH}]^{+}(24)$ and $176\left[\mathrm{M}-2 \mathrm{CH}_{3}\right]^{+}$ (11); Analysis: calcd. \%C, 64.08; \%H, 4.83; \%O, 31.88. Found: $\% \mathrm{C} 64.12 ; \% \mathrm{H}, 4.81 ; \% \mathrm{O} 31.09$.

\subsection{2,2-Dimethyl-2H-chromene-5,7,8(6H)-trione (7)}

Compound 7 was obtained by the same procedure described for the synthesis of compound 4 as faint brown crystals $\left(0.59 \mathrm{~g}, 40 \%\right.$ yield) with $\mathrm{mp} 187-189^{\circ} \mathrm{C}$; IR $(\mathrm{KBr}) v_{\max } \mathrm{cm}^{-1}: 1,750,1,720,1,711$, 1,581, 1,345, 1,099, 901, 869; ${ }^{1} \mathrm{H}-\mathrm{NMR}\left(\mathrm{CDCl}_{3}\right): \delta 6.82(\mathrm{Ar}-1 \mathrm{H}, \mathrm{d}, J=9.2 \mathrm{~Hz}, \mathrm{H}-4), 5.84(\mathrm{Ar}-1 \mathrm{H}, \mathrm{d}$, $J=8.2 \mathrm{~Hz}, \mathrm{H}-3), 4.07$ (Ar-1H, s, H-6), 1.44(6H, s, 2CH$) ;{ }^{13} \mathrm{C}-\mathrm{NMR}\left(\mathrm{CDCl}_{3}\right): \delta 176.5(\mathrm{C}-8), 196.2$ (C-5,7), 141.2 (C-8a), 117.4 (C-4a), 126.5 (C-3), 115.5 (C-4), 45.8(C-6),86.7 (C-2), $29.5\left(2 \mathrm{CH}_{3}\right.$, C-9,10); EIMS $\left(\mathrm{C}_{11} \mathrm{H}_{10} \mathrm{O}_{4}\right)$ (\% int.) $\mathrm{m} / z 206(100), 178[\mathrm{M}-\mathrm{CO}]^{+}(34)$ and $176\left[\mathrm{M}-2 \mathrm{CH}_{3}\right]^{+}$(27); Analysis: calcd. \%C, 64.06; \%H, 4.88; \%O, 31.04. Found: \%C 63.92; \%H, 5.81; \%O 30.89.

\subsection{4,4,8,8-Tetramethyl-4,8-dihydro-2H-dipyrano-benzo-1,2-dione (8)}

Compound $\mathbf{8}$ was obtained by the same procedures described for the synthesis of compounds $\mathbf{2}$ and 3, respectively, by refluxing compound $7(0.01 \mathrm{~mol})$ with 3 -chloro-3-methylbut-1-yne $(0.01 \mathrm{~mol})$, followed by ring closure to give the di pyrano derivative in dry xylene for $8 \mathrm{~h}$, then the precipitate obtained was filtered off and crystallized from a mixture of cyclohexane and ethanol give compound $\mathbf{8}$ as faint brown crystals $\left(0.85 \mathrm{~g}, 40 \%\right.$ yields) with $\mathrm{mp} 292-294{ }^{\circ} \mathrm{C}$; UV (MeOH) $\lambda_{\max }: 462(\log \varepsilon 3.75)$, 302 (log $\varepsilon$ 3.97), $271(\log \varepsilon 4.47), 261(\log \varepsilon 4.43)$; IR (KBr) $v_{\max } \mathrm{cm}^{-1}: 1,789,1,754,1,598,1,375$, 1,049, 911, 859; ${ }^{1} \mathrm{H}-\mathrm{NMR}\left(\mathrm{CDCl}_{3}\right): \delta 6.75$ (d, $J=8.2$, Ar-1H, H-10), 6.48 (d, $\left.J=8.3 \mathrm{~Hz}, \mathrm{Ar}-1 \mathrm{H}, \mathrm{H}-6\right)$, 5.89 (d, $J=9.2 \mathrm{~Hz}, \mathrm{Ar}-1 \mathrm{H}, \mathrm{H}-9), 5.84(\mathrm{Ar}-1 \mathrm{H}, \mathrm{d}, \mathrm{H}-5), 1.34\left(12 \mathrm{H}, \mathrm{s}, 4 \mathrm{CH}_{3}\right) ;{ }^{13} \mathrm{C}-\mathrm{NMR}\left(\mathrm{CDCl}_{3}\right)$ :

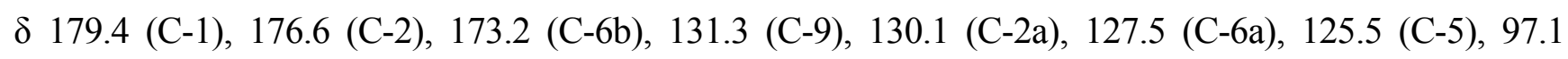
(C-10a), 88.9 (C-8), 88.5 (C-4), $29.5\left(2 \mathrm{CH}_{3}, \mathrm{C}-13,14\right), 28.7\left(2 \mathrm{CH}_{3} ; \mathrm{C}-11,12\right)$; EIMS $\left(\mathrm{C}_{16} \mathrm{H}_{16} \mathrm{O}_{4}\right)$ (\% int.) $m / z 272(100), 257\left[\mathrm{M}-1 \mathrm{CH}_{3}\right]^{+}(31), 244[\mathrm{M}-\mathrm{CO}]^{+}(17)$ and $242\left[\mathrm{M}-2 \mathrm{CH}_{3}\right]^{+}$(14); Analysis: calcd. $\% \mathrm{C}, 70.54 ; \% \mathrm{H}, 5.95 ; \% \mathrm{O}, 23.50$. Found: $\% \mathrm{C} 70.32 ; \% \mathrm{H}, 5.81 ; \% \mathrm{O} 23.39$.

\subsection{6,6,10,10-Tetramethyl-6,10-dihydro-2H-dipyrano[2,3-f:2',3'-h]chromen-2-one (10)}

Wittig reaction was carried out as described in the discussion [6] using 0.01 mol of compound 8 . $30 \%$ Aqueous $\mathrm{NaOH}$ solution $(30 \mathrm{~mL})$ was added to the Wittig reaction product and the mixture was 
boiled for $3 \mathrm{~h}$, cooled, acidified with ice cold hydrochloric acid, and the reaction mixture refluxed for another $2 \mathrm{~h}$. The precipitate obtained after cooling was filtered off, washed with water and crystallized from dil. ethanol to give compound 10 through the intermediate 9 as pale brown crystals $(1.24 \mathrm{~g}, 50 \%$ yield) with mp 295-296 ${ }^{\circ} \mathrm{C}$; UV (MeOH) $\lambda_{\max }: 211(\log \varepsilon$ 4.27), $282(\log \varepsilon 3.75)$ and $232(\log \varepsilon$ 3.97); IR (KBr) $v_{\max } \mathrm{cm}^{-1}: 1,720,1,711,1,591,1,345,1,089,901,869 ;{ }^{1} \mathrm{H}-\mathrm{NMR}\left(\mathrm{CDCl}_{3}\right): \delta 7.75(\mathrm{Ar}-1 \mathrm{H}, \mathrm{d}$, $J=8.95 \mathrm{~Hz}, \mathrm{H}-4), 6.88$ (Ar-1H, d, $J=8.9 \mathrm{~Hz}, \mathrm{H}-12), 6.84$ (Ar-1H, d, $J=9.2 \mathrm{~Hz}, \mathrm{H}-8), 5.97$ (Ar-1H, s, H-3,11), 1.84 (3H, s, H-13,14,15,16); ${ }^{13} \mathrm{C}-\mathrm{NMR}\left(\mathrm{CDCl}_{3}\right)$ : 151.9 (C-12b), 149.8 (C-8b), 148.9 (C-4b), 139.5 (C-4), 128.5 (C-11), 128.2 (C-7,12), 116.4 (C-8), 113.6 (C-3), 108.2 (C-8a), 105.3 (C-4a), 102.5 (C-12a), 86.5 (C-6,10), $28.6\left(4 \mathrm{CH}_{3}\right.$;C-13,14,15,16) as lit. [6]; EIMS $\left(\mathrm{C}_{19} \mathrm{H}_{18} \mathrm{O}_{4}\right)(\%$ int.) $\mathrm{m} / \mathrm{z}$ 310. (100), $292[\mathrm{M}-\mathrm{CO}]^{+}(32), 285\left[\mathrm{M}-1 \mathrm{CH}_{3}\right]^{+}(21)$ and $250\left[\mathrm{M}-4 \mathrm{CH}_{3}\right]^{+}$(7); Analysis: calcd. \%C, 73.54; \%H, 5.85; \%O, 20.64. Found: \%C 73.32; \%H, 5.81; \%O 20.29.

\subsection{Biological Activity}

\subsubsection{Experimental Animals}

Young adult (30 $\pm 5 \mathrm{~g}$ ) ICR mice (half male and half female) were provided by the Egyptian Holding Company for Biological Products and Vaccines, Cairo, Egypt. Animals were maintained under standard conditions of ventilation, temperature $\left(25 \pm 2{ }^{\circ} \mathrm{C}\right)$, humidity $(60 \%-70 \%)$ and light/dark condition (12/12 h). The rats were housed in stainless steel cages and provided with free access to food and drinking water ad libitum.

\subsubsection{Effect of Compound $\mathbf{1 0}$ on the Tumor of S180-Bearing Mice}

The effect of compound $\mathbf{1 0}$ solution on tumor growth was estimated by evaluating body weight, tumor weight, and percentage of tumor inhibition. S180 tumor cell line was originally obtained from Cairo Institute of Oncology, Cairo, Egypt and maintained as the ascites form by serial passages intraperitoneal in ICR mice. For solid tumor development, S180 cell suspension $\left(0.2 \mathrm{~mL}, 2 \times 10^{7}\right.$ cells $\left./ \mathrm{mL}\right)$ was inoculated subcutaneously into right armpits of mice under sterile condition. The mice were divided into six random groups (10 in each): S180-bearing control, normal control, compound 10 $(50,100,250$ and $500 \mathrm{mg} / \mathrm{kg}$ body weight). Test doses were decided on the basis of findings from preliminary studies. Body weight of animals was recorded before the experiment. The doses administrated p.o. daily for 12 days. Normal control and S180-bearing control groups received the same volume of normal saline. On the 13th day, all animals were euthanized. Their body and tumor weights were obtained and documented [11].

3.10.3. Assessment of Humoral Immune Function: Quantitative Hemolysis of Sheep Red Blood Cells (QHS) Assay

The mice were injected i.p. with 3:5 (v/v) sheep red blood cells (SRBC, $0.2 \mathrm{~mL}$ ) prepared in normal saline on the 8th day of the experiment. QHS assay was performed in those animals following the immunization. Eyeballs were removed and single cell suspensions of $1 \times 10^{6} / \mathrm{mL}$ were prepared in phosphate buffer solution (PBS). A total of $1.0 \mathrm{~mL}$ of $0.4 \% \mathrm{SRBC}$ and $1.0 \mathrm{~mL}$ of $10 \%$ guinea pig 
serum were mixed with cell suspension and incubated for $1 \mathrm{~h}$ at $37^{\circ} \mathrm{C}$. After a 3 min centrifugation at $3,000 \mathrm{rpm}$, the absorbance of the supernatant was measured at $413 \mathrm{~nm}$ using a spectrophotometer [12].

\subsubsection{Assessment of Cellular Immune Function}

For the assessment of cellular immune function, lymphocyte proliferation and Natural Killer (NK) cell cytotoxicity tests were performed. After the experiment was completed, their spleens were aseptically removed and filtered over a double layer of stainless-steel mesh to obtain single cell suspension. After these washes in Hanks' balanced salt solution, the spleen cells were finally suspended in 10\% FCS RPMI 1640 media supplemented with benzyl penicillin $100 \mathrm{U} / \mathrm{mL}$, streptomycin $100 \mu \mathrm{g} / \mathrm{mL}$. The cell number was adjusted to $3 \times 10^{6}$ cells $/ \mathrm{mL}$ of culture media for subsequent experiments [12].

\subsubsection{Measurement of Lymphocyte Proliferation}

For the splenocyte proliferation assay, the spleen cell suspension was added to micro plate wells with $5 \mu \mathrm{g} / \mathrm{mL}$ of concanavalin A (Con A, from Canavalia ensiformis Type III, Sigma, Munich, Germany) and a polyclonal $\mathrm{T}$ cell mitogen. The micro plates were cultured at $37{ }^{\circ} \mathrm{C}$ for $72 \mathrm{~h}$ in the humidified 5\% $\mathrm{CO}_{2}$ incubator. At $72 \mathrm{~h}, 1 \mu \mathrm{Ci} /$ well ${ }^{3} \mathrm{H}-\mathrm{TdR}$ (thymidine, [methl- ${ }^{3} \mathrm{H}$ ]) was added to each well. The cells were harvested $16 \mathrm{~h}$ later and the radioactivity incorporated was counted using a liquid scintillation counter [12].

\subsubsection{Evaluation of NK Cell Cytotoxicity}

The splenocyte prepared as described above were used as effector cells. YAC-1 cells, mice lymphoma sensitive to NK cells were used as target cells. Effectors and target cells resuspended in RPMI-1640 medium supplemented with 3\% heat-inactivated fetal bovine serum were added to each well of a 96-well U-bottom micro culture plate in triplicate to obtain an effectors/target (E/T) ratio of 50:1, and incubated at $37{ }^{\circ} \mathrm{C}$ in the humidified $5 \% \mathrm{CO}_{2}$ incubator for $8 \mathrm{~h}$. After centrifugation, the culture supernatants were admixed with lactate dehydrogenase (LDH) solution (100 $\mu \mathrm{L} /$ well) and the amount of released LDH was determined. The OD value of each well was measured at $490 \mathrm{~nm}$ using a spectrophotometer. The percentage of cytotoxicity generated by NK cells was calculated according to the following formula:

$$
\left[\text { Cytotoxicity }(\%)=\frac{\mathrm{OD}_{\mathrm{er}}-\mathrm{OD}_{\mathrm{esr}}-\mathrm{OD}_{\mathrm{tsr}}}{\mathrm{OD}_{\mathrm{tmr}}-\mathrm{OD}_{\mathrm{tsr}}} \times 100\right]
$$

where $\mathrm{OD}_{\text {er }}\left(\mathrm{OD}_{\text {experimental release }}\right)$ was the $\mathrm{LDH}$ release from co-cultures at an $\mathrm{E} / \mathrm{T}$ ratio of 50:1; $\mathrm{OD}_{\text {esr }}$ $\left(\mathrm{OD}_{\text {effector spontaneous release }}\right)$ and $\mathrm{OD}_{\text {tsr }}\left(\mathrm{OD}_{\text {target spontaneous release }}\right)$ were spontaneous $\mathrm{LDH}$ releases from effector and target cells incubated with medium alone, respectively; and $\mathrm{OD}_{\mathrm{tmr}}\left(\mathrm{OD}_{\text {target maximum release }}\right)$ was the maximum release from target cells lysed with the lysis solution [13]. 
3.10.4.3. Assessment of Nonspecific Immune Function: Phagocytic Activity of Macrophage

Phagocytic activity of macrophages was used to assess the nonspecific immune function. The mice

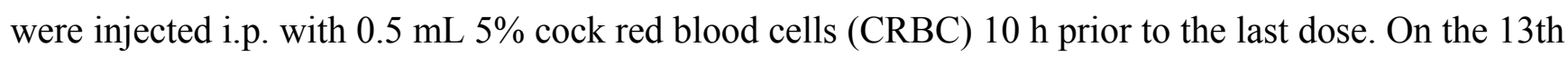
day of the experiment macrophages were obtained from the peritoneal exudates harvested by peritoneal lavage using sterile cold Hanks' solution. The number of CRBC ingested by macrophages was counted in an optical microscope [12].

\subsection{Statistical Analysis}

Data were expressed as the mean \pm standard deviation (S.D.) in tables. Statistical analyses were carried out using the analysis of variance (ANOVA) and post-hoc tests for multiple comparisons. Differences were considered statistically significant at $p<0.05$ [14].

\section{Conclusions}

In summary we have developed a new simple route approach to naturally dipetalolactone starting from resorcinol and substituted-1-butyne via Fremy's salt as mediator oxidizing agent and examined its effect on in vivo Immunomodulatory activities in S180-bearing mice, where higher concentrations of compound $\mathbf{1 0}$ appeared to show more effective inhibition of tumor by increasing the antibodies secreted by spleen cells in mice.

\section{Acknowledgments}

We thank staff of Faculty of Sciences Aristotle University of Thessaloniki, Greece for the performance of some 1D and 2D NMR and MS spectral analyses experiments.

\section{Conflicts of Interest}

The authors declare no conflict of interest.

\section{References}

1. Robert, D.; Murray, H. The Naturally Occurring Coumarins; Fortschitte Der Chemie Organischer Naturstoffe: Glasgow, UK, 2002; Volume 38, pp. 1-619.

2. Kiran, D.P.; Swati, P.J.; Sunil, R.B.; Shubhada, R.T. Pattern of anti-HIV dipyranocoumarin expression in callus cultures of Calophyllum inophyllum Linn. J. Biotechnol. 2007, 130, 346-353.

3. Murray, R.D.H.; Méndez, J.; Brown, S.A. The Natural Coumarins. Occurrence. In Chemistry and Biochemistry; John Wiley \& Sons Ltd: New York, NY, USA, 1982; pp. 343-345.

4. Ribeiro, C.V.C.; Kaplan, M.A.C. Evolutionary families of trends producers coumarins in angiosperms. Quim. Nova 2002, 25, 533-538.

5. Melo, M.F.F.; Zickel, C.S. Genres zanthoxylum L. and Esenbeckia Kunth (Rutaceae) in the State of Pernambuco, Brazil. Acta Bot. Bras. 2004, 18, 73-90.

6. Francis, F.; Alexander, I.G.; Roger, R.D.; Peter, G.W. Detalolactone: A novel pyrano coumarin from the root bark of Zanthoxylum dipetalum. Phytochemistry 1976, 15, 313-316. 
7. Patai, S.; Rappoport, Z. The Chemistry of the Quinoid Compounds; Wiley-Interscience: New York, NY, USA, 1988.

8. Tisler, M. Heterocyclic Quinones. In Advances in Heterocyclic Chemistry; Katritzky, A.R., Ed.; Academic Press: London, UK, 1989; Volume 45, p. 37.

9. McOmie, J.F.W.; Blatchly, J.M. The THIELE-Winter Acetoxylation of Quinones. In Organic Reactions; Wiley: New York, NY, USA, 1972; Volume 19, p. 199.

10. Villemin, D.; Bar, N.; Hammadi, M. Triflic acid an efficient catalyst for the Thiele-Winter reaction. Tetrahedron Lett. 1997, 38, 4777-4778.

11. Doherty, N.S. Selective effects of immuno-suppressive agents against the delayed hyper sensitivity response and humoral response to sheep red blood cells in mice. Agents Action 1981, 11, 237-242.

12. Nelson, D.S.; Mildenhall, P. Immuno-modulatory activity of Madhuca longifolia. Aust. J. Exp. Biol. Med. Sci. 1967, 45, 113-130.

13. Jang, Y.Y.; Cho, D.; Kim, S.K.; Shin, D.J.; Park, M.H.; Lee, J.J.; Chin, M.G.; Shin, J.H.; Suh, H.P.; Ryang, D.W. An improved flow cytometry-based natural killer cytotoxicity assay involving calcein AM staining of effector cells. Ann. Clin. Lab. Sci. 2012, 42, 42-49.

14. Sokal, R.P.; Rohlf, F.J. The Principles and Practice of Statistics in Biological Research, 1st ed.; Freeman WH: San Francisco, CA, USA, 1969; pp. 469-484.

Sample Availability: Samples of the compounds $\mathbf{6 , 7 , 8 , 9}$ and $\mathbf{1 0}$ are available from the authors.

(C) 2013 by the authors; licensee MDPI, Basel, Switzerland. This article is an open access article distributed under the terms and conditions of the Creative Commons Attribution license (http://creativecommons.org/licenses/by/3.0/). 